\title{
Técnica de un colgajo: una nueva alternativa en el tratamiento de las fisuras palatinas
}

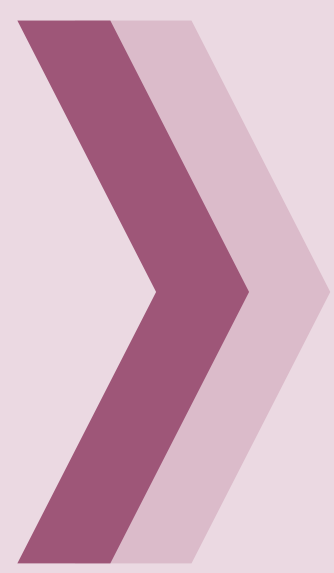

\author{
A flap technique: a new \\ alternative in the treatment \\ palate clefts
}

\section{Percy Rossell-Perry ${ }^{1}$ Omar Cotrina-Rabanal ${ }^{2}$}

1. Médico cirujano plástico. Director médico Programa Lima Outreach Surgical Center. Fundación Interplast EE UU.

2. Odontólogo. Director médico Fundación Armonizar, Lima, Perú.

\section{RESUMEN}

INTROducción. Diferentes técnicas quirúrgicas para el tratamiento de las fisuras palatinas han sido descritas hasta la actualidad mostrando buenos y malos resultados. La técnica de dos colgajos (Bardach) es la técnica más utilizada en Estados Unidos, sin embargo, esta presenta algunas limitaciones. OBлetivo. Presentar los resultados quirúrgicos obtenidos en el tratamiento de las fisuras palatinas unilaterales con una nueva técnica quirúrgica: la palatoplastia de un colgajo. Material y método. El presente es un estudio descriptivo. Se describe una nueva técnica quirúrgica utilizada desde el año 2007 por nosotros y los resultados quirúrgicos obtenidos de 316 pacientes con fisura unilateral completa fueron operados con la técnica de un colgajo entre los años 2007 al 2014. Resultados. El cierre anatómico de los paladares fisurados operados se obtuvo en un $94.31 \%$ de los casos y el cierre funcional se observó en un $96.84 \%$ de los casos utilizando la técnica propuesta. Se observó un numero de 12 casos que evolucionaron con fistulas palatinas (3.79\%). Otros malos resultados y complicaciones son presentados en este estudio. Conclusiones. Se pudo observar un cierre anatómico y funcional aceptable de las fisuras palatinas con la utilización de la nueva técnica presentada. Los hallazgos observados avalan los aportes propuestos en este artículo con la nueva técnica quirúrgica para el tratamiento de la fisura palatina unilateral.

Palabras clave. Cirugía plástica, fisura del paladar.

\section{SUMMARY}

InTRODUction. Different surgical techniques for cleft palate repair have been described actually illustrating good and bad results. The two flap palatoplasty (Bardach) is the most used surgical technique in USA however it has some limitations. OBJECTIVE. Compare the surgical results obtained in the unilateral cleft palate management using a new surgical technique: the one flap palatoplasty MateRIAL AND METHOD. This is a descriptive study. We describe a new surgical technique used since 2007 by us and the obtained surgical outcomes 316 patients with complete unilateral cleft palate were operated using the one flap technique between 2007 to 2014. Results. Anatomical closure of the cleft palates have been obtained in 94.31 $\%$ of the cases and functional closure have been observed in $96.84 \%$ of the cases using the proposed technique. We observed 12 patients with palatal fistula (3.79\%) in this study. Other bad results and complications are presented in this paper. Conclusion. We observed an acceptable anatomical and functional closure of the cleft palates using the presented surgical technique. These findings support our concepts presented in this paper using the new surgical technique for unilateral cleft lip repair.

KeY woRDs. Surgery, Plastic, Cleft palate. 


\section{INTRODUCCIÓN}

La fisura palatina (FP) es una patología congénita frecuente en Perú. Afecta áreas importantes del desarrollo del individuo como es el habla y la alimentación.

Los protocolos quirúrgicos varían mucho según las instituciones. La tendencia general es la reparación de las diferentes formas de FP con una sola técnica.

En un estudio realizado en EE UU por Katzel, ${ }^{1}$ se realizó un censo en 803 cirujanos miembros de la Academia Americana de Cirugía Plástica y se observó que la técnica más utilizada fue la de Bardach (denominada también como técnica de los dos colgajos) en combinación con la técnica de veloplastia intravelar (45\%), seguida muy cerca por la técnica de Furlow (42\%).

La técnica de dos colgajos tiene una serie de limitaciones, entre ellas las siguientes:

- Utilización de incisiones relajantes en ambos segmentos palatinos, que deja áreas cruentas laterales.

- Una mayor probabilidad de desarrollar hipoplasia maxilar secundaria a la cirugía primaria palatina. ${ }^{2-4}$

$\Delta$ Limitación para tratar formas graves de FP, con esta técnica se observa una mayor proporción de fístulas palatinas. ${ }^{5-6}$

Esta situación llevó a la creación de una técnica que permita tratar las FP con mejores resultados.

En el periodo de 2007 a 2014, se empleó una nueva técnica quirúrgica denominada de un colgajo, que permite corregir los casos de FP con menor número de incisiones y menor disección quirúrgica. Figura 1.

\section{MATERIAL Y MÉTODO}

Estudio descriptivo sobre un total de 316 pacientes con FP unilateral completa no sindrómica operados entre los años 2007 y 2014, mediante la utilización de la técnica quirúrgica de un colgajo.Los pacientes fueron operados en las clínicas Los Andes de Lima y la Clínica Santa Teresa de Abancay y en los hospitales Manuel Núñez Butrón de Puno, Regional de Huaraz, Perú y Regional de Puerto Maldonado.
La técnica de un colgajo consiste en la utilización de un solo colgajo mucoperióstico, en lugar de dos como se hace en la técnica convencional, para los casos de FP unilateral. Figura 1.

Los resultados del tratamiento aplicado por estos protocolos fueron evaluados de manera retrospectiva a través del examen físico de los pacientes. Así se determinó la presencia o no de fístulas palatinas en los paladares operados con esta técnica. Estas fístulas fueron evaluadas al menos un mes después de la cirugía de paladar.

Se define como fístula palatina a la presencia de comunicación entre la nariz y la cavidad oral a través del paladar duro o del blando luego de la palatoplastia primaria.

Las fístulas anteriores fueron excluidas, ya que la fisura alveolar se opera de manera secundaria entre los siete y nueve años.

\section{Técnica quirúrgica}

El paciente es colocado en posición supina, con el cuello extendido. Luego de la intubación, unos cinco minutos antes de iniciar la cirugía, se coloca el abrebocas de Dingman y se procede a la infiltración con anestésico local. Las incisiones quirúrgicas se hacen con una hoja de bisturí 15 . Figura 1.

La cirugía se inicia en la región posterior (uvular) con la técnica de uvuloplastia unilateral que preserva una hemiúvula. ${ }^{7}$ Figura $1 \mathrm{~A}$.

Luego, se realiza la incisión con el bisturí en los bordes de la fisura, en ambos lados. En el lado no fisurado, con el electrocauterio, se completa el diseño del colgajo mucoperióstico en continuidad con la incisión anterior y, luego, medialmente sobre la gingiva siguiendo la línea de erupción dental tal como lo recomienda Cartens. ${ }^{8}$ Esta incisión, que permitirá extender lateralmente el diámetro del colgajo a utilizar, termina $1 \mathrm{~cm}$ sobre el paladar blando, para evitar tensión en el cierre medial. Figura 1A. A continuación, es elevado el colgajo diseñado en el plano subperióstico.

Una disección cuidadosa se debe realizar alrededor del pedículo vascular, para facilitar la movilización del colgajo. Figura 1B. 


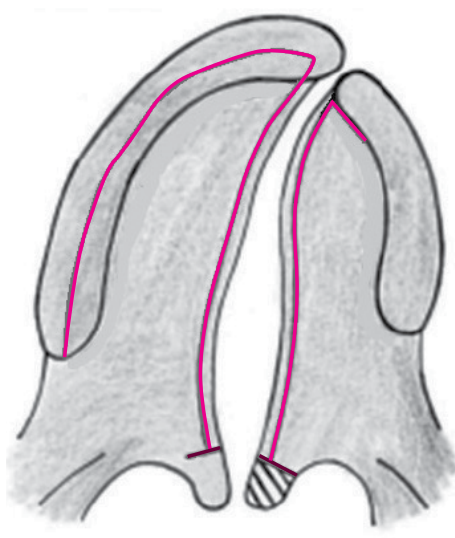

A

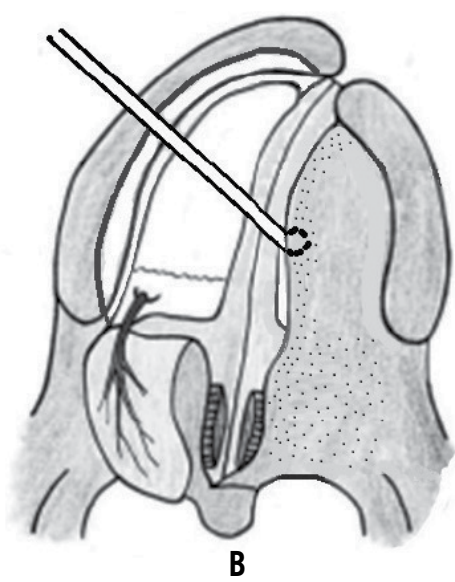

B

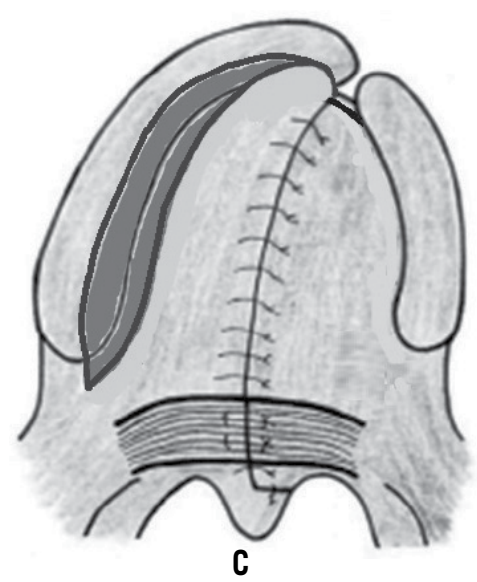

Figura 1. Técnica quirúrgica de palatoplastia de un colgajo en el tratamiento de la fisura palatina unilateral.

Una pequeña incisión es necesaria anteriormente en el lado fisurado a nivel gingivomucoso para facilitar la aproximación anterior de la mucosa en este segmento. Luego, a través de la incisión del borde medial de la fisura en el lado fisurado, se realiza una pequeña disección de la mucosa en el plano subperióstico (paladar duro) y submucoso (paladar blando), para facilitar el cierre en la línea media con el colgajo del lado no fisurado. Figura $1 B$.

Una vez realizada la disección se realiza el cierre de la úvula, usando la técnica de uvuloplastia unilateral publicada por Rossell-Perry et al.? Figura $1 C$.

Luego, se realiza el cierre del plano mucoso nasal, con suturas reabsorbibles de calibre 5/0. Esto permitirá llevar a cabo el reparo del plano muscular (veloplastia intravelar) tal como lo recomienda Sommerlad. ${ }^{9}$

Finalmente, se hace el cierre de la mucosa oral, con suturas reabsorbibles de calibre $5 / 0$. Si no se puede cerrar con esta sutura se requerirá de una mayor disección de los tejidos, para disminuir la tensión del cierre, y, así, evitar la formación de fístulas. Figuras $1 \mathrm{C}$ y 2.

\section{RESULTADOS}

El estudio incluyó 316 pacientes, 209 de sexo masculino y 107 de sexo femenino, con la edad de un año al momento de la cirugía.

El cierre anatómico de los paladares fisurados operados se obtuvo en $94,31 \%$ de los casos y el cierre funcional se observó en $96,84 \%$ de los casos.

Hubo $29,43 \%$ de resultados no deseados y complicaciones. Los principales fueron hipertermia (34 casos; $10,75 \%$ ) y sangrado en el postoperatorio (16 casos; $5,06 \%$ ). Tabla 1.

Los resultados obtenidos con la técnica de un colgajo en dos casos de FP unilateral moderada, se muestran en las Figuras 2 y 3.

\section{DISCUSIÓN}

A fines del siglo pasado y en la primera década del presente, para tratar todas las formas de FP, se utilizó la técnica de Bardach de dos colgajos ${ }^{10}$ asociada a la veloplastia intravelar de Sommerlad, ${ }^{9}$ con resultados variables y con $15 \%$ de fístulas palatinas. ${ }^{11}$ En EE UU, más de $90 \%$ de los cirujanos utilizan una sola técnica quirúrgica (sea Bardach o Furlow) para tratar las diferentes formas de 
FP. ${ }^{1}$ Esta situación implica la utilización de más incisiones y disección quirúrgica que las necesarias en las formas de FP más leves. Esto se podría reflejar en una mayor afección del crecimiento maxilar, descrito por otros autores, ${ }^{2-4}$ aunque esta hipótesis requiere de una investigación adicional para que pueda ser confirmada. Los autores de este artículo en las FP usan la técnica de Bardach solo en $12 \%$ de los casos y la de Furlow en $10 \% .{ }^{11}$

En Europa, los protocolos de atención promueven la utilización de dos tiempos quirúrgicos para reducir la posibilidad de afección del crecimiento maxilar, sin embargo, diferentes estudios muestran una mayor probabilidad de afección del habla y de fístulas palatinas. ${ }^{7,12,13}$ Además, estas técnicas demandan un seguimiento del paciente para garantizar que se pueda completar el segundo tiempo quirúrgico, situación que es difícil de cumplir en zonas de nivel socioeconómico bajo, donde muchos pacientes se operan en campañas quirúrgicas.

La nueva técnica quirúrgica basada en un colgajo e indicada en las FP unilaterales permite obtener un cierre anatómico adecuado y no se observa mayor número de fístulas palatinas $(3,48 \%)$ en comparación con los estudios publicados (de $0 \%$ a $25 \%$ ). ${ }^{14-19}$ Su uso se limita a las FP unilateral leve y moderada, de acuerdo a la clasificación basada en el índice palatino publicada por Rossell-Perry et al. ${ }^{6}$ Las formas graves de FP unilaterales son tratadas en dos tiempos quirúrgicos y el cierre diferido del paladar duro se realiza seis meses después, con la técnica de un colgajo.

Es importante mencionar que técnicas similares han sido descritas anteriormente. Así, Gillet y Clarke ${ }^{20}$ han descrito una serie de casos usando una combinación de las técnicas de Von Langenbeck y de Bardach, en búsqueda de minimizar las incisiones. Otra publicación, hecha por Fudalej, ${ }_{1}^{21}$ describe el uso de un colgajo mucoperióstico del lado no fisurado a manera de colgajo bipediculado (Von Langenbeck). Sin embargo, esta técnica a diferencia de la nuestra, sigue utilizando incisiones en el lado fisurado.

En relación a los resultados no deseados obtenidos, la complicación más común observada fue la hipertermia $(10,75 \%)$. Este evento es común en cirugías de FP, se presenta durante las primeras 48 horas del postoperatorio, es de duración corta (no más de 24 horas) y no mayor de 38 grados. Duffy ${ }^{22}$ ha relacionado este evento con dos mecanismos causales, el incremento de la producción de calor y la disminución de la pérdida de calor.

El acto quirúrgico es responsable del incremento de la producción de calor por la acción de pirógenos liberados desde el lugar de injuria quirúrgica. Los mecanismos que interfieren con la pérdida de calor son comunes de observar en el postoperatorio, así hay una vasoconstricción periférica, la cual directamente disminuye la disipación del calor. También se sabe que está en relación con el déficit en la hidratación del paciente y no con proceso infeccioso alguno. De ahí que se recomienda disminuir en lo posible el periodo de ayuno preoperatorio. El tratamiento, en este caso, consiste en hidratación adecuada del paciente y el uso de fármacos antipiréticos de ser necesarios. Otras series reportan un porcentaje variable de hipertermia postoperatoria, Zhan et al. recientemente observaron $0,80 \%$ de hipertermia, ${ }^{23}$ sin embargo, esta complicación podría estar subestimada.

El sangrado postoperatorio, presente en 5,06\% de los casos, fue de frecuencia baja debido al uso de una sonda Foley. ${ }^{24}$

El porcentaje de dehiscencias (parciales y totales) también fue bajo $(2,21 \%)$. Este resultado es importante de considerar, pues la tensión del cierre podría ser una de las principales observaciones al uso de un solo colgajo. El porcentaje de fístulas y dehiscencias observado indica que esta técnica permite un cierre anatómico sin mayor riesgo de aparición de fístulas palatinas.

El cierre funcional de los paladares operados estimado a través del número de casos con insuficiencia velofaríngea (hipernasalidad) moderada a grave $(3,16 \%)$ es también aceptable y comparable a los resultados mostrados por otros estudios. Así, Sommerlad, con su técnica de veloplastia intravelar, reporta $5 \%$ de insuficiencia velofaríngea y La Rossa, $6 \%{ }^{9,25}$ Cabe mencionar que la insuficiencia velofaríngea es más frecuente en las FP con un mayor grado de hipoplasia (FP bilaterales e incompletas). 
Tabla 1. Malos resultados y complicaciones

\begin{tabular}{lrr} 
& $\mathrm{n}$ & $\%$ \\
$\Delta$ Hipertemia* $^{\star}$ Sangrado postoperatorio* $^{*}$ & 34 & 10,75 \\
$\Delta$ Fístula $_{\Delta}$ Insuficiencia velofaringea & 16 & 5,06 \\
$\Delta$ Dehiscencia parcial & 11 & 3,48 \\
$\Delta$ Dehiscencia total & 10 & 3,16 \\
$\Delta$ Edema lingual & 5 & 1,58 \\
$\Delta$ Infección & 2 & 0,63 \\
$\Delta$ Otros & 2 & 0,63 \\
\hline Total & 1 & 0,31 \\
& 12 & 3,79 \\
\hline
\end{tabular}
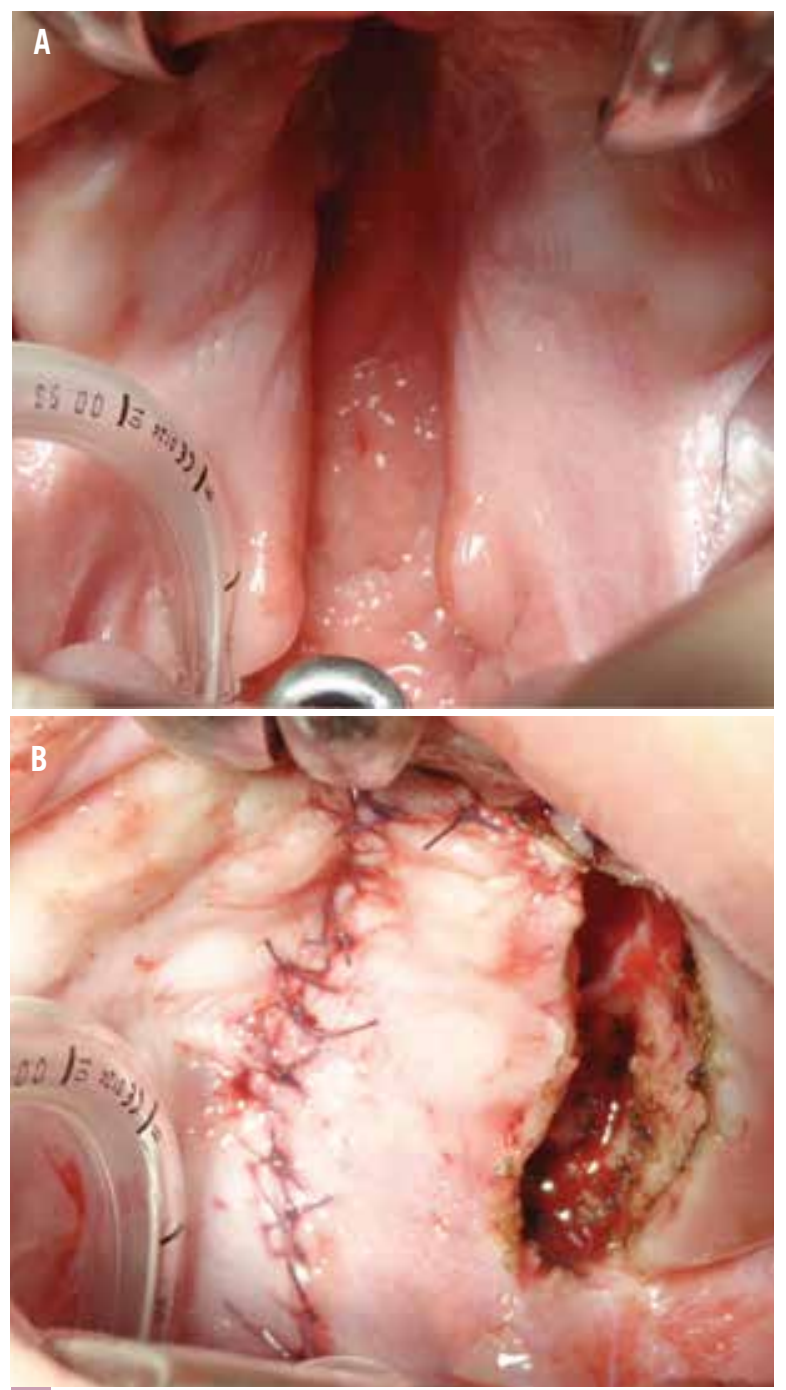

Figura 2. Fisura palatina unilateral moderada. A. Preoperatorio. B. Resultado postoperatorio inmediato con utilización de la técnica de un colgajo.
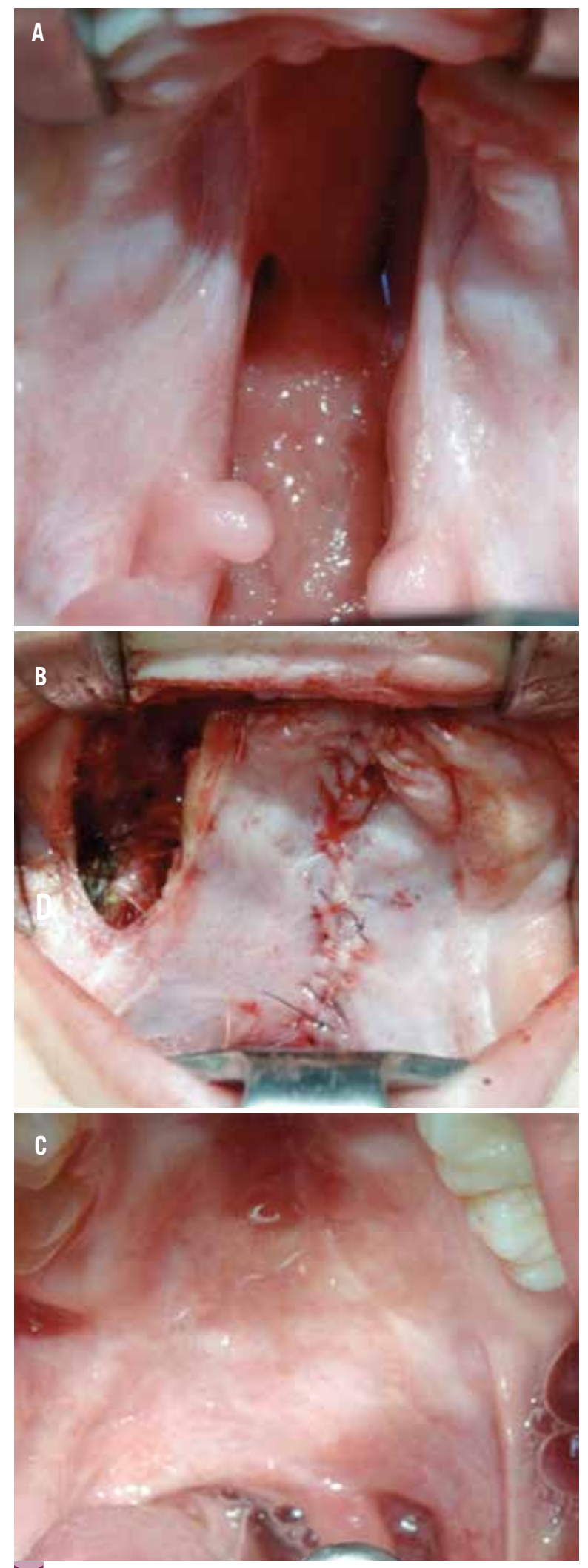

Figura 3. Fisura palatina unilateral moderada. A. Preoperatorio. B. Resultado postoperatorio inmediato con utilización de la técnica de un colgajo. C. Resultado postoperatorio a los seis años de edad. 
Dado que el presente estudio se ha desarrollado en FP unilaterales que tienen un mejor pronóstico, esto podría explicar las diferencias observadas con otros estudios.

La técnica de un colgajo puede ser utilizada también en el manejo de fístulas leves a moderadas localizadas en el paladar duro y como segundo tiempo quirúrgico en las cirugías de cierre diferido del paladar duro. Las ventajas de esta técnica sobre el crecimiento del maxilar superior serán estudiadas en una investigación posterior.

\section{CONCLUSIONES}

La técnica de un colgajo es un procedimiento seguro, predecible y que permite obtener un cierre anatómico y funcional en las fisuras palatinas unilaterales con menores incisiones y tiempos quirúrgicos que otras técnicas convencionales.

\section{REFERENCIAS BIBLIOGRÁFICAS}

I. Katzel E, Basile P, Koltz P, Marcus J. Current surgical practices in cleft care: cleft palate repair techniques and postoperative care. Plast. Reconstr Surg. 2009; I24(3):899-906.

2. Yu-Fang Liao, I-Ying Yang, Wang R, Yun C, Chiung-Shing Huang. Twostage palate repair with delayed hard palate closure is related to favorable maxillary growth in unilateral cleft lip and palate. Plast Reconstr Surg. 2010;125(5):1503-10.

3. Liao YF, Mars M. Long-term effects of palate repair on craniofacial morphology in patients with unilateral cleft lip and palate. Cleft Palate Craniofac J. 2005;42:594-600.

4. Liao YF, Cole TJ, Mars M. Hard palate repair timing and facial growth in unilateral cleft lip and palate: A longitudinal study. Cleft Palate Craniofac J. 2006;43:547-556.

5. Rossell-Perry P. Nueva clasificación de severidad de fisuras labiopalatinas. Act Méd Per. 2006;23(2):59-66.

6. Rossell-Perry P, Caceres-Nano E, Gavino-Gutierrez A. Association between palatal index and cleft palate repair outcomes in patients with complete unilateral cleft lip and palate. JAMA Facial Plastic Surgery. 2014;16(3):206-210.

7. Rossell-Perry P, Navarro-Gasparetto C, Caceres-Nano E, CotrinaRabanal O.A prospective, randomized double-blind clinical trial study to evaluate a method for uvular repair during primary palatoplasty.J Plast Surg Hand Surg. 2014;48(2): 132-5.

8. Carstens M. Sequential cleft management with the sliding sulcus technique and alveolar extension palatoplasty. J Craniofac Surg. 1999;10:503-18.

9. Sommerlad B.A technique for cleft palate repair. Plast Reconstr Surg. 2003; I I2(6): 1542-48.

10. Bardach J. Two flap palatoplasty: Bardach's technique. Operative techniques in plastic and reconstructive surgery. 1995; 2(4): 2 I I-2 I4.

II. Rossell-Perry P. Tratamiento de la fisura labio palatina. Lima: Universidad San Marcos; 2009.

12. Witzel MA, Salyer KE, Ross RB. Delayed hard palate closure: The philosophy revisited. Cleft Palate J. 1984;21:263-269.

13. Noordhoff MS, Kuo J, Wang H, Witzel MA. Development of

\section{Correspondencia}

Dr. Percy Rossel Perry

prossell3p@hotmail.com

Fecha de recepción: 4 de junio de 2014

Fecha de aceptación: 23 de junio de 2014 articulation before delayed hard palate closure in children with cleft palate: a cross sectional study. Plast Recons Surg. 1987;80: 51 8-524.

14. Hollier L. Cleft palate and velopharyngeal incompetence. Selected Readings Plast Surg. 1997;8:I.

15. Berkman MD. Early non-surgical closure of postoperative palatal fistulae. Plast Reconstr Surg. 1978;62:537.

16. Abyholm FE, Borchgrevink HHC, Eskeland G. Palatal fistulae following cleft palate surgery. Scand J Plast Reconstr Surg. 1979; 13:295.

17. Amaratunga NA. Occurrence of oronasal fistulas in operated cleft palate patients. J Oral Maxillofac Surg. 1988;46:834.

18. Cohen SR, Kalinowski J, La Rossa D, Randall P. Cleft palate fistulas: A multivariate statistical analysis of prevalence, etiology, and surgical management. Plast Reconstr Surg. 1991;87:1041.

19. Emory RE Jr., Clay RP, Bite U, Jackson I.T. Fistula formation and repair after palatal closure: An institutional perspective. Plast. Reconstr Surg. 1997;99:1535.

20. Gillett DA, Clarke HM.The hybrid palatoplasty:A preliminary report. Can J Plast Surg. 1996;4:157-160.

2I. Fudalej P, Katsaros C, Dudkiewicz Z, Bergé S, et al. Cephalometric outcome of two types of palatoplasty in complete unilateral cleft palate. Br J Oral Maxillofac Surg. 2013; 5 I (2): I44-8

22. Duffy M. Fever following palatoplasty: An evaluation based on fever volume. Plast Reconstr Surg. 1966;38(I):32-35.

23. Zhang Z, Fang S, Zhang Q, Chen L, et al. Analysis of complications in primary cleft lips and palates surgery. J Craniofac Surg. 2014; 25(3):968-71.

24. Rossell-Perry P, Schneider W, Gavino-Gutierrez A. A comparative study to evaluate a simple method for the management of postoperative bleeding following palatoplasty. Arch Plast Surg. 20I3;40(3):263-266.

25. La Rossa D, Hunenko O, Kirschner R, Low D, et al. The Children's Hospital of Philadelphia modification of the Furlow double opposing z plasty: Long term speech and growth results. Clin Plast Surg. 2004;31:243-249.

\section{Conflictos de interés}

El autor declara no tener conflictos de interés durante el planteamiento, ejecución de la investigación y la elaboración del artículo para su publicación. 